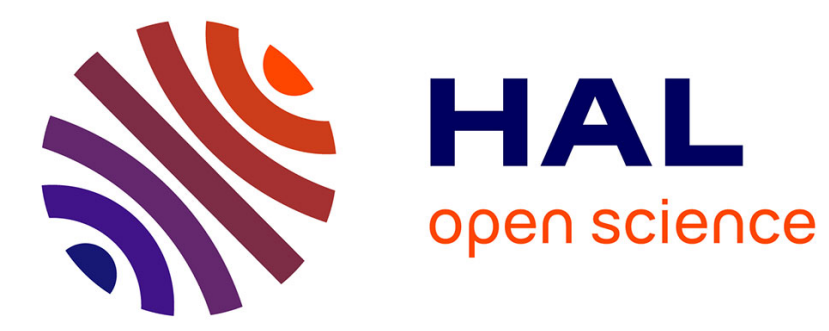

\title{
RECENT RESULTS IN THE THEORETICAL DESCRIPTION OF CL AND EBIC DEFECT CONTRASTS
}

S. Hildebrandt, J. Schreiber, W. Hergert

\section{- To cite this version:}

S. Hildebrandt, J. Schreiber, W. Hergert. RECENT RESULTS IN THE THEORETICAL DESCRIPTION OF CL AND EBIC DEFECT CONTRASTS. Journal de Physique IV Proceedings, 1991, 01 (C6), pp.C6-39-C6-44. 10.1051/jp4:1991607 . jpa-00250692

HAL Id: jpa-00250692

https://hal.science/jpa-00250692

Submitted on 1 Jan 1991

HAL is a multi-disciplinary open access archive for the deposit and dissemination of scientific research documents, whether they are published or not. The documents may come from teaching and research institutions in France or abroad, or from public or private research centers.
L'archive ouverte pluridisciplinaire HAL, est destinée au dépôt et à la diffusion de documents scientifiques de niveau recherche, publiés ou non, émanant des établissements d'enseignement et de recherche français ou étrangers, des laboratoires publics ou privés. 


\title{
RECENT RESULTS IN THE THEORETICAL DISCRIPTION OF CL AND EBIC DEFECT CONTRASTS
}

\author{
S. HILDEBRANDT, J. SCHREIBER and W. HERGERT \\ Martin-Luther-Universität Halle-Wittenberg, Fachbereich Physik, Friedemann-Bach-Platz \\ 6, D-4020 Halle (Saale), Germany
}

\begin{abstract}
The theoretical description used for the analysis of beam-voltage dependent CL and EBIC contrasts from a surface-parallel dislocation is summarized. The importance of the use of a realistic carrier generation model is discussed and suitable methods of the evaluation of defect parameters (depth position, defect strength) are proposed.
\end{abstract}

\section{1.- Introduction.}

The availability of a comprehensive theoretical model is of great importance for the accurate analysis of defect contrasts in the cathodoluminescence (CL) and electron beam induced current (EBIC) mode of the scanning electron microscope (SEM). In order to enable a convenient and routine processing of experimental contrast data, this model should be as simple as possible but still universal and accurate enough for an exact derivation of relevant parameters such as the depth position of the single defect and its defect strength which describes the local recombination activity in the semiconductor.

The literature of the past decade contains various models of the description of defect contrasts. Considering the widely accepted volume recombination model, basic works by DONOLATO [1,2] and PASEMANN [3,4] performed an analytical calculation of the EBIC contrast profile from a surfaceparallel dislocation in first-order approximation of the carrier density or by application of the perturbation theory. In the same way, PASEMANN and HERGERT [5,6] calculated the CL contrast, too. This concept, which is also the starting point for our considerations uses a uniform sphere generation that is only a rough approximation of the real carrier generation distribution which makes this model questionable for practical use. On the other hand, in the last years also realistic generation functions were integrated into the contrast calculation by Monte Carlo simulation [7] or numerical solution of the continuity equation [8]. These fully numerical models, however, are still too expensive and timeconsuming when performed on a laboratory PC if defect parameters are to be derived by non-linear regression or interactive graphical fit. In this context, the evaluation of the contrast profile area [9-11] is an interesting alternative but leaves some open questions behind, for instance concerning the beamvoltage dependence of the contrast half-width.

In the present paper the EBIC and CL contrasts are recalculated basing on the analytical model mentioned above but now using a realistic carrier generation for the computation of the carrier distribution which is performed partly by numerical integration.

Considering the dependence of the contrast on the accelerating voltage of the electron beam, the new results are compared with the sphere generation and consequences for the analysis of experimental results are discussed which include the evaluation of the contrast profile area as well as the EBIC to CL contrast ratio. We restrict ourselves to the basic defect configuration of a single dislocation parallel to the sample 
surface with infinite surface recombination velocity, where the collecting barrier for EBIC (Schottky contact) is located.

\section{2.- Theoretical model.}

In Fig. 1 the geometrical configuration of the profile measurement and the volume recombination model for a surface-parallel dislocation is shown. The dislocation is represented by cylindrical regions centered in the depth $\mathbf{z}_{d}$ where the total and radiative carrier lifetimes have values $\tau$ 'and $\tau_{r}$ 'different from those of the defect free matrix region $\left(\tau, \tau_{r}\right)$.

The defect contrast profile is defined by

$$
C(\xi)=\frac{I(\xi)-I_{0}}{I_{0}}
$$

where $\mathrm{I}, \mathrm{I}_{0}$ are the EBIC or CL signals at the defect or in the matrix, respectively. The contrast profile area $\mathrm{A}(\xi)$ is obtained by integration:

$$
A=\int_{-\infty}^{\infty} d \xi c(\xi)
$$

The EBIC contrast is given by [3]

$$
c_{E B I C}=-\frac{\tau / \tau^{\prime}-1}{\tau I_{0}^{E B I C}} \int_{Q_{d}} d^{3} r j_{E B I C}(z) p_{0}(r)
$$
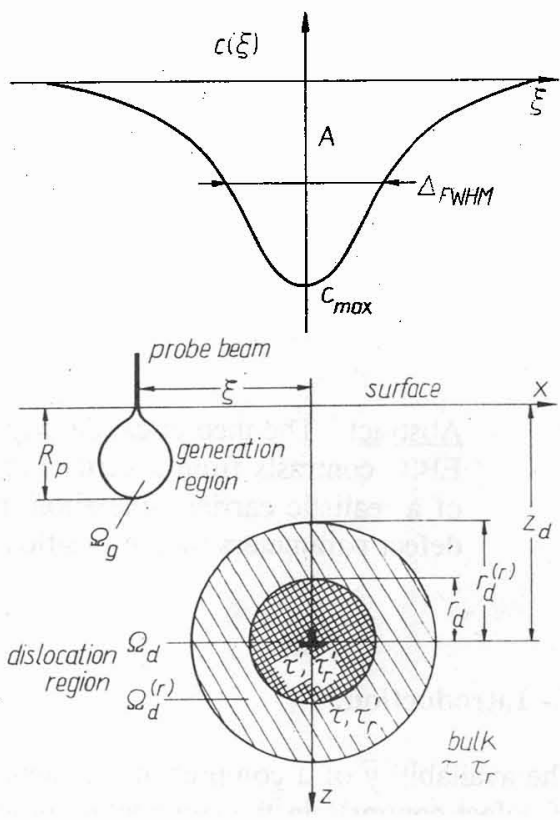

(3) Fig. 1. Geometrical configuration of the surfaceparallel dislocation in the sample

and the CL contrast was calculated to be [12]

$$
c_{C L}=-\frac{\tau / \tau^{\prime}-1}{\tau_{r} I_{0}^{C L}} \int_{Q_{d}} d^{3} r j_{C L}(z) p_{0}(r)+\frac{\tau_{r} / \tau_{r}^{\prime}-1}{\tau_{r} I_{0}^{C L}} \int_{Q_{d}^{(r)}} d^{3} r A^{\prime}(z) p_{0}(r)
$$

Here, $j_{\text {EBIC }}, j_{C L}$ are the matrix signals for unit point source excitation at depth $z . A^{\prime}(z)$ is an optical loss function. Due to the use of the first-order approximation, $p_{0}(r)$ is the minority carrier density in the defect-free semiconductor. Problems, which are connected with the application of the perturbation theory, are beyond the scope of this paper (see e.g. [13]).

First of all, the matrix signals $\mathrm{I}_{0}{ }^{\mathrm{EBIC}}, \mathrm{I}_{0}{ }^{\mathrm{CL}}$ can be most easily calculated by means of our unified description of luminescence and induced current signals developed in $[14,15]$ for a realistic generation as well as the generation sphere or the point source $\left(j_{E B I C}, j_{C L}\right)$.

The central problem consists in the calculation of the carrier density $\mathrm{p}_{0}(\mathbf{r})$. It is given in [16] for the case of uniform sphere generation analytically. The integrals in (3), (4) can be written as

$$
\int_{\Omega_{d}} d^{3} r f(z) p_{0}(r)=\iint_{f_{d}} d x d z f(z) Q_{0 y}(x, z)
$$

$\left(f_{d}\right.$ - dislocation cross section area) with the y-integrated carrier density 


$$
Q_{0 y}(x, z)=\int_{-\infty}^{\infty} d y p_{0}(r)
$$

For sphere generation, (6) can also be solved analytically [12].

To obtain a solution for a realistic generation, the generalized generation function by KOCH, WERNER and OELGART $[17,18]$ was selected. This function exhibits the shape

$$
g(r)=G_{0} g_{r}(r) g_{z}(z)
$$

with radial and depth distribution

$$
g_{r}(r)=\sum_{i=1}^{2} a_{i} \exp \left[-\left(\frac{r}{a_{i}}\right)^{2}\right], g_{z}(z)=\sum_{j=1}^{2} b_{j} \exp \left[-\left(\frac{z-z_{j}}{\eta_{j}}\right)^{2}\right],\left(z_{2}=0\right)
$$

Knowing $\mathbf{g}(\mathbf{r})$, the carrier density is given by the method of Green's function $G\left(\mathbf{r}, \mathbf{r}^{\prime}\right)$ for the semi-infinite sample. The performance of some of the integrations in $(6),(8)$ finally leads to the equation

$$
\begin{aligned}
Q_{0 y}(x, z)=\frac{1}{2 \sqrt{\pi} D} \sum_{i=1}^{2} & a_{i} \sigma_{i} \sum_{j=1}^{2} b_{j} \int_{0}^{\infty} d z^{\prime} \exp \left[-\left(\frac{z^{\prime}-z_{j}}{\eta_{j}}\right)^{2}\right] \int_{-\infty}^{\infty} d x^{\prime} \exp \left[-\left(\frac{x^{\prime}}{\sigma_{i}}\right)^{2}\right] * \\
& *\left[K_{0}\left(\frac{\sqrt{\left(x-x^{\prime}-\xi\right)^{2}+\left(z-z^{\prime}\right)^{2}}}{L}\right)-K_{0}\left(\frac{\sqrt{\left.x-x^{\prime}-\xi\right)^{2}+\left(z+z^{\prime}\right)^{2}}}{L}\right)\right]
\end{aligned}
$$

(D - diffusion coefficient, $\mathrm{L}$ - diffusion length, $\mathrm{K}_{0}$ - modified Bessel function of 2 nd kind and Oth order). In the calculation of the EBIC and CL contrasts, this double integral is solved numerically.

In (5) still the cross-section integration remains. In the case of $r_{d}$ \& $L$ the integrand can be approximated by its central point value at $\left(\xi, z_{d}\right)$ without affecting the shape of the contrast profile.

Thus, the calculation of the CL and EBIC contrast from a surface-parallel dislocation in the case of realistic generation is complete. To summarize, as an example for the EBIC contrast we get in this way

$$
c_{E B I C}(\xi)=-\lambda \frac{j_{E B I C}\left(z_{d}\right)}{I_{0}^{E B I C}} D Q_{0 y}\left(\xi, z_{d}\right)
$$

The defect strength $\lambda$ appears as a prefactor in the EBIC contrast.

In the second term of the CL contrast (4) a "radiative" defect strength $\lambda_{r}$ appears. In the first-order approximation it is

$$
\lambda^{(1)}=\pi\left(\frac{r_{d}}{L}\right)^{2}\left(\frac{\tau}{\tau^{\prime}}-1\right), \lambda_{r}^{(1)}=\pi\left(\frac{r_{d}^{(r)}}{L}\right)^{2}\left(\frac{\tau_{r}}{\tau_{r}^{\prime}}-1\right)
$$

The EBIC contrast profile area

$$
A_{E B I C}=-\frac{G_{0}\left(\tau / \tau^{\prime}-1\right)}{4 \pi L^{2} I_{0}^{E B I C}} \int_{f_{d}} d x d z j_{E B I C}(z) \int_{z_{T}}^{\infty} d z^{\prime} g_{z}\left(z^{\prime}\right) G_{1}\left(z, z^{\prime}\right)
$$

is a significantly simpler expression where in comparison to the contrast formula the $y$-integrated carrier density is replaced by 


$$
\int_{z_{T}}^{\infty} d z^{\prime} g_{z}\left(z^{\prime}\right) G_{1}\left(z, z^{\prime}\right)=2 \pi L\left\{e^{-\left(z-z_{)}\right) / L}\left[\Phi\left(-\frac{1}{L}, z_{T}, z\right)-\Phi\left(\frac{1}{L}, z_{T},\right)\right]+\Phi\left(\frac{1}{L}, z, \infty\right)\right\}
$$

$\left(G_{1}\right.$ - one-dimensional Green's function [14]). This integral can be written in terms of the universal function

$$
\Phi\left(x, z_{1}, z_{2}\right)=\int_{z_{1}}^{z_{2}} d z g_{z}(z) e^{-x\left(z-z_{1}\right)}
$$

introduced in $[14,15]$ for the unified description of the signals from homogeneous, defect-free regions. Since the function $\Phi$ is analytically known for various generation rates $g_{z}(z)$, equation (12) makes the calculation of the EBIC and CL contrast profile area very convenient for a fast contrast analysis to obtain the defect strength $\lambda$. Furthermore, in (13) the occurence of a surface dead layer $z_{T}$ (e.g. depletion layer or metal contact) is explicitly regarded for the first time.

To derive the defect strengths from the contrast, generally the depth position $z_{d}$ of the dislocation has to be known. Here, the analysis of $c_{E B I C} / c_{C L}$ is recommended. PASEMANN, HERGERT [5] obtained for the contrast ratio

$$
\frac{c_{E B I C}}{c_{C L}}\left(z_{d}\right)=\frac{\frac{I_{0}^{C L}}{\tau / \tau_{r}} j_{E B I C}\left(z_{d}\right)}{I_{0}^{E B I C} j_{C L}\left(z_{d}\right)}
$$

Since this formula contains only quantities of the defect-free semiconductor, the application of a realistic generation and the inclusion of surface layers is easily performed.

\section{3.- Examples and discussion.}

In this section, examples for the beam voltage dependence of the contrast are discussed since EBIC and CL experiments are often carried out in this way. Fig. 2 a shows the EBIC contrast $c_{E B I C}\left(U_{b}, \xi=0\right)$ from surface-parallel dislocations located at different depths. In contrast to the realistic generation function, the use of the generation sphere leads to completely different results (Fig. $2 \mathrm{~b}$ ). We recognize that in reality no contrast extremum occures as it is followed from the sphere generation model. Its application is possible only at low beam voltages when the electron range is low compared to $\mathrm{z}_{\mathrm{d}}$ [4].

EBIC controst

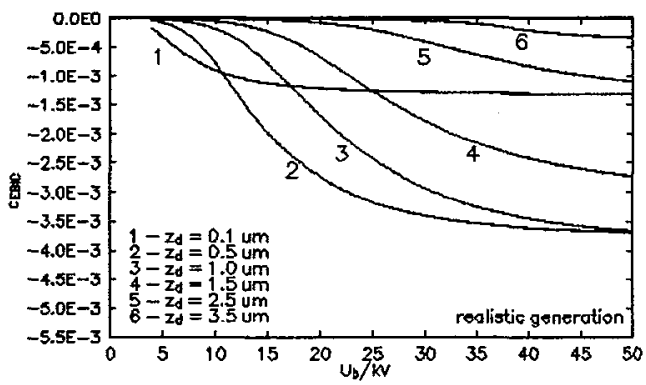

Fig. 2 a. EBIC contrast $(\xi=0, L=1 \mu \mathrm{m}, \lambda=0.01$ $\pi, \mathrm{GaAs})$
EBIC contrast

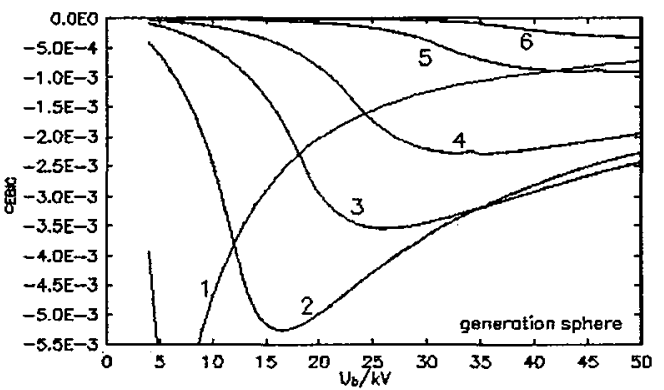

Fig. 2 b. EBIC contrast (same parameters as Fig. 2 a)

Looking at the CL contrast (Fig. 3), the correspondence of the two models is still poorer already at small $\mathrm{U}_{\mathrm{b}}$. By use of a realistic generation, an estimate of the dislocation depth should be better possible as 
CL controst

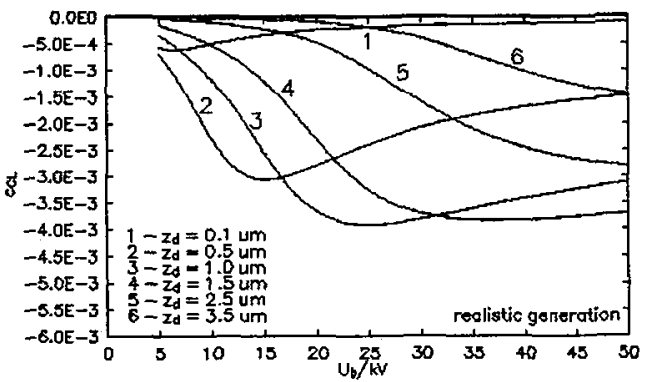

Fig. 3 a. CL contrast (same parameters as Fig. $2 ; \alpha=$ $0.75 \mathrm{\mu m}^{-1}, \lambda_{\mathrm{r}}=0$ )

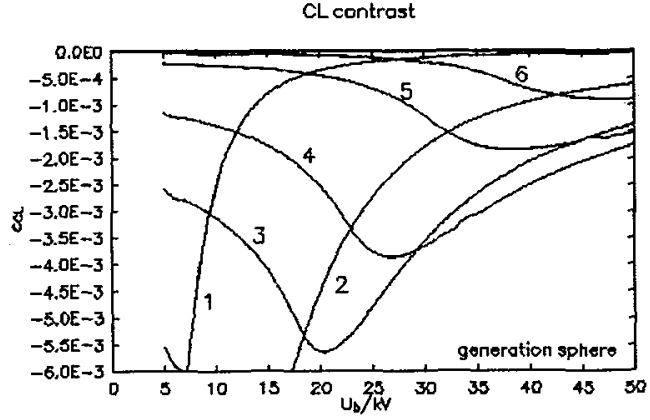

Fig. 3 b. CL contrast (same parameters as Fig. 3 a)

from the EBIC contrast at least for $z_{d}<2 \mu \mathrm{m}$. However, if both EBIC and CL contrasts are measured, a much more accurate and simple procedure is the calculation of the contrast ratio (14) (see Fig. 6).

It should be noted that the beam voltage dependence of the EBIC and CL contrast for realistic generation looks very similar to that of the corresponding contrast profile area shown in [10] so that no further examples will be given here.

FWHM of EBIC and CL contrast profiles

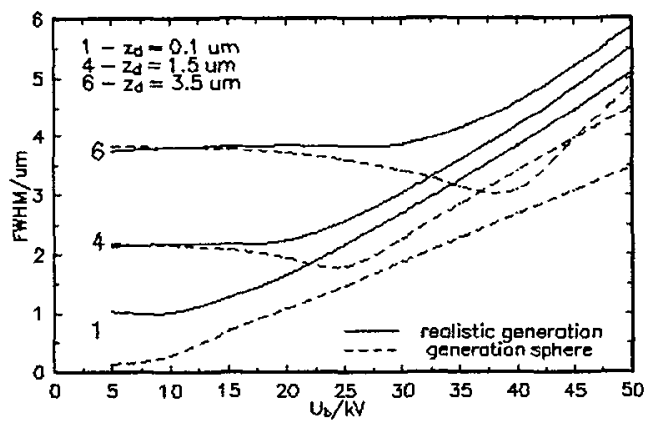

Fig. 4. Contrast profile half-width (same parameters as previous figures)

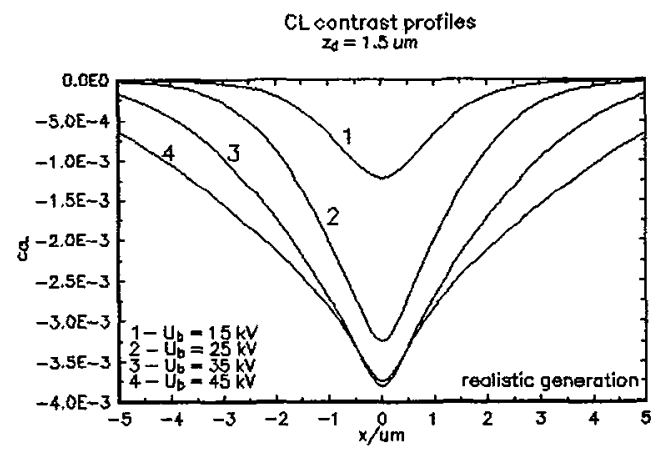

Fig. 5. CL contrast profiles (same parameters as Fig. 3 a) width of the contrast profiles. From Fig. 4 one can see that at higher beam voltages (depending on $z_{d}$ ) the real FWHM is larger than obtained with the sphere model. Especially, the contrast half-width no longer exhibits a minimum. So there is no optimum resolution of the EBIC image as it had been deduced from the early findings of DONOLATO [19] which correspond to the dashed curves of Fig. 4.

The calculation of the contrast profiles leads to interesting results (Fig. 5). At low beam voltages the contrast is of a Gaussian-similar shape but with increasing $U_{b}$ it broadens and exhibits a sharper peak. At $45 \mathrm{kV}$ the contrast profile is of nearly triangular form with an indication of a narrower tip at the centre. In this voltage range the contrast profile already reflects the two Gaussian parts of the radial carrier generation distribution (7).

Finally, Fig. 6 shows the EBIC to CL contrast ratio for the Schottky contact geometry. The slope of the curves is strongly determined by the dislocation depth $z_{d}$, but the value of the absorption coefficient $\alpha$ also influences the contrast ratio. For the evaluation of $z_{d}$ from the contrast ratio, in principle an experiment at one fixed beam voltage is sufficient. The analysis of the beam-voltage dependence of $\mathrm{c}_{\mathrm{EBIC}} / \mathrm{c}_{\mathrm{CL}}$, however, should yield more accurate results. The matrix parameters $\mathrm{L}$ and $\alpha$ are deduced from the $U_{b}$-dependent $I_{0}$ signals, too. 


\section{4.- Conclusions.}

The results presented in this paper emphasize the importance of the application of a realistic generation function in the contrast calculations. In order to test the validity of the model for the analysis of the specific defect investigated the performance of beamvoltage dependent combined EBIC and CL experiments is favoured. Then the defect relevant parameters $\lambda, \mathbf{z}_{d}, \ldots$ can be deduced with sufficient reliability and high effectiveness with the present theoretical description. In any case, the limits of our approach (surface-parallel dislocation configuration, convergence of the perturbation series) have to be regarded.

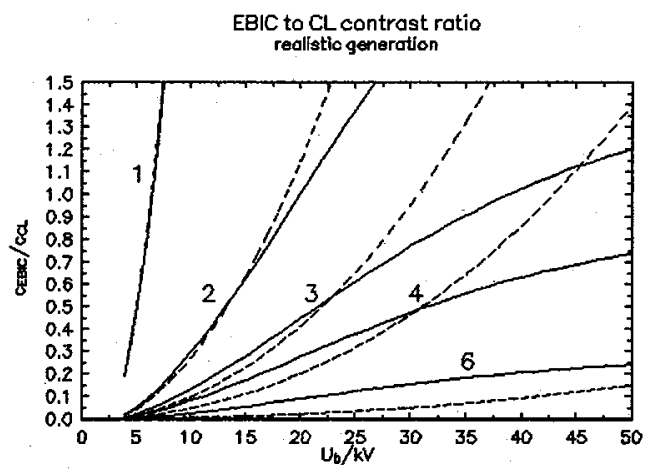

Fig. 6. EBIC to CL contrast ratio (same parameters as Fig. $3 ; \mathrm{z}_{\mathrm{T}}=0.05 \mu \mathrm{m} ; \alpha=0.75 \mu \mathrm{m}^{-1}$ - solid lines, $\alpha$ $=0$ - dashed lines)

\section{References.}

[1] DONOLATO,C, phys. stat: sol. (a) 66 (1981) 445

[2] DONOLATO,C, J. Physique Colloq: 44 (1983) C4-289

[3] PASEMANN, L, Ultramicroscopy 6 (1981) 237

[4] PASEMANN, L, BLUMTRITT, H, GLEICHMANN, R, phys. stat. sol. (a) 70 (1982) 197

[5] PASEMANN, L, HERGERT, W, Ultramicroscopy 19 (1986) 15

[6] PASEMANN, L, HERGERT, W, Izv. Akademii Nauk SSSR, Ser. Fiz. 51 (1987) 1528

[7] CZYZEWSKI, Z, JOY, D C, Scanning 12 (1990) 5

[8] WEBER, G, DIETRICH, S, HÜHNE, M, ALEXANDER, H, Inst. Phys. Conf. Ser. 100 (1989) 749

[9] DONOLATO, C, BIANCONI, M, phys. stat. sol. (a) 102 (1987) K 7

[10] HILDEBRANDT, S, HERGERT, W, phys. stat. sol. (a) 119 (1990) 689

[11] SCHREIBER, J, HILDEBRANDT, $S$, this volume

[12] HERGERT, $W$, Thesis B, Halle 1988

[13] HERGERT, W, PASEMANN, L, HILDEBRANDT, $S$, this volume

[14] HERGERT, W, HILDEBRANDT, S, PASEMANN, L, phys. stat. sol. (a) 102 (1987) 819

[15] HERGERT, $W$, this volume

[16] HERGERT, W, PASEMANN, L, phys. stat. sol. (a) 85 (1984) 641

[17] KOCH, F, Thesis, Berlin 1987

[18] WERNER, U, KOCH, F, OELGART, G, J. Phys. D 21 (1988) 116

[19] DONOLATO, C, Optik $52(1978 / 79) 19$ 